\title{
van der Waals density functionals built upon the electron-gas tradition: Facing the challenge of competing interactions
}

\author{
Kristian Berland, ${ }^{1,2}$ Calvin A. Arter, ${ }^{3}$ Valentino R. Cooper, ${ }^{4}$ Kyuho Lee, ${ }^{2,5}$ \\ Bengt I. Lundqvist, ${ }^{6}$ Elsebeth Schröder, ${ }^{1}$ T. Thonhauser, ${ }^{3}$ and Per Hyldgaard ${ }^{1}$ \\ ${ }^{1}$ Microtechnology and Nanoscience, MC2, Chalmers University of Technology, SE-412 96 Göteborg, Sweden \\ ${ }^{2}$ Molecular Foundry, Lawrence Berkeley National Laboratory, Berkeley, California 94720, USA \\ ${ }^{3}$ Department of Physics, Wake Forest University, Winston-Salem, North Carolina 27109, USA \\ ${ }^{4}$ Materials Science and Technology Division, Oak Ridge National Laboratory, \\ Oak Ridge, Tennessee 37831-6114, USA \\ ${ }^{5}$ Department of Chemical and Biomolecular Engineering, University of California, Berkeley, \\ California 94720, USA \\ ${ }^{6}$ Department of Applied Physics, Chalmers University of Technology, SE-412 96 Göteborg, Sweden
}

(Received 7 January 2014; accepted 3 April 2014; published online 28 April 2014)

\begin{abstract}
The theoretical description of sparse matter attracts much interest, in particular for those ground-state properties that can be described by density functional theory. One proposed approach, the van der Waals density functional (vdW-DF) method, rests on strong physical foundations and offers simple yet accurate and robust functionals. A very recent functional within this method called vdW-DF-cx [K. Berland and P. Hyldgaard, Phys. Rev. B 89, 035412 (2014)] stands out in its attempt to use an exchange energy derived from the same plasmon-based theory from which the nonlocal correlation energy was derived. Encouraged by its good performance for solids, layered materials, and aromatic molecules, we apply it to several systems that are characterized by competing interactions. These include the ferroelectric response in $\mathrm{PbTiO}_{3}$, the adsorption of small molecules within metal-organic frameworks, the graphite/diamond phase transition, and the adsorption of an aromatic-molecule on the $\operatorname{Ag}(111)$ surface. Our results indicate that vdW-DF-cx is overall well suited to tackle these challenging systems. In addition to being a competitive density functional for sparse matter, the vdWDF-cx construction presents a more robust general-purpose functional that could be applied to a range of materials problems with a variety of competing interactions. (C) 2014 AIP Publishing LLC. [http://dx.doi.org/10.1063/1.4871731]
\end{abstract}

\section{INTRODUCTION}

Sparse matter is important and calls for a theoretical description as accurate as one for dense matter. Despite the urgency of this need there were surprisingly few density functional theory (DFT) papers accounting for the interactions in sparse media before the 1990s. Such interactions may be weak and of several kinds, but it is the delicate balance between them that is key to determining materials properties. Since the 90s, there has been an upsurge in electronstructure calculations describing nonlocal correlations, in particular van der Waals (vdW) forces. A spectrum of methods have been proposed. Some rely on semi-empirical pair potentials to correct semilocal generalized gradient approximations (GGA), ${ }^{1,2}$ while others are fully nonlocal correlation functionals. ${ }^{3-5}$ Among them the van der Waals-density functional (vdW-DF) ${ }^{3,6,7}$ method stands out in its high ambitions of designing a tractable functional with strong physical foundations, thereby resulting in low computational demands and high accuracy. With this in mind, early vdW-DF method development ${ }^{3,6-15}$ focused on constructing a nonlocal correlation functional that was (i) nonempirical, that is, entirely given by the ground-state density with no external parameters, and (ii) robust, thereby creating a framework for the inclusion of dispersive or vdW interactions ${ }^{16,17}$ and, more generally, truly nonlocal correlations. Such a method should cover different energy scales. These functionals build upon the experience obtained during decades of use of their predecessors, the local-density approximation ${ }^{18-20}$ (LDA) and the constraint-based formulations of the GGAs. ${ }^{21-29}$

Initially, relatively little attention was devoted to exchange. The "modest" goal of the pioneering vdW-DF1 functional ${ }^{3}$ was to describe vdW bonds with separations typical for them, 3-4 $\AA$, and to ensure that there was no unphysical binding from the exchange-only description. ${ }^{3,13,15}$ For these reasons, the revised Perdew, Burke and Ernzerhof (revPBE) exchange functional ${ }^{30}$ was chosen. Numerous applications of vdW-DF1, however, implicated this choice of exchange as the cause of a consistent overestimation of intermolecular separation distances and lattice constants. Subsequently, the focus turned to the development of appropriate exchange functionals to pair with the nonlocal correlation term. ${ }^{31-35}$ One strategy was to adjust existing exchange functionals such that the interaction energies of a subset of molecular systems agreed with those of advanced quantumchemical calculations. ${ }^{36}$

This paper sums up results obtained with a more rigorously derived exchange functional for the vdW-DF nonlocal correlation; one designed with a critical eye on consistency between exchange and correlation. This exchange functional follows a tradition of describing the fully interacting and therefore screened electron gas primarily in terms of the 
plasmon-response behavior. ${ }^{18,22,23,37-43}$ Ingredients are the adiabatic connection formula ${ }^{19,22,23}$ (ACF) and the assumption that a (single-)plasmon-pole approximation ${ }^{3,11,12,44}$ for the dielectric function, $\epsilon$, can be picked to represent the full exchange-correlation (XC) energy in the spirit of the electrongas tradition. ${ }^{19,22,23,41-43}$ Further details are given in the Appendix.

By "electron gas tradition" we mean that specific conservation laws are adhered to; including having an $\mathrm{XC}$ hole that contains just one charge unit, and physical constraints built into the plasmon-pole response ${ }^{3,6}$ that describes the nonlocal correlations. The recent most explicit functionals, vdW$\mathrm{DF} 1^{3,6}$ and vdW-DF2, ${ }^{5}$ expand beyond the local electron-gas in terms of a plasmon propagator $S=1-1 / \epsilon$ and arrive at a nonlocal correlation energy, $E_{\mathrm{c}}^{\mathrm{nl}}[n]$, that is quadratic in the density $n(\mathbf{r})$. The underlying plasmon-pole basis still reflects a collectivity that captures broader density variations. ${ }^{3,12,44-46}$ Seamless integration in the homogeneous electron gas (HEG) limit and the observance of physical constraints ${ }^{3,19,22,23,44,47}$ are features that vdW-DF shares with LDA and GGA. The inner exchange functional that describes the plasmon response giving rise to the nonlocal correlations in vdW-DF1 is also based on many-body theory in a diagrammatic form which was used in the design of early GGAs. ${ }^{25}$ vdW-DF2 is instead based on scaling laws for atoms and thus designed for higher accuracy for atoms and small molecules. Two of us have recently designed an exchange functional LV-PW86r that extends the vdW-DF1 track. The LV-PW86r exchange closely follows the vdW-DF1 inner functional up to moderate density variations, as described by the dimensionless parameter $s \propto|\nabla n| / n^{4 / 3}$, while switching to a better motivated and tested exchange description at large $s$ values. ${ }^{35}$ Paired with the vdW-DF1 correlation, we obtain an improved consistency between the exchange and correlation description and therefore we label the full functional vdW-DF-cx, where cx emphasizes the aim of using a consistent exchange description.

In Secs. II-V, we examine the efficacy of a set of nonempirical vdW-DF versions. Specifically, we examine the recently developed vdW-DF-cx and its precursor C09x. We aim to demonstrate that these functionals are capable of not only describing molecular problems but also are general-purpose materials theory tools. These nonlocal functionals have an approximative conservation of the XC hole. The vdW-DF-cx goes furthest in enforcing consistency between exchange and correlation and leverages the conservation of the full $\mathrm{XC}$ hole instead of, for example, seeking to conserve the exchange hole separately from the nonlocal correlation. Such conservation suggests good transferability.

\section{TOWARDS A GENERAL-PURPOSE DENSITY FUNCTIONAL}

\section{A. Plasmon theory of the electron gas}

A general-purpose density functional should be able to describe both molecular interactions and the binding within bulk materials. This has been a long-standing objective for the development of nonlocal functionals by $\mathrm{us}^{3,6-15,48}$ and others. ${ }^{49}$ While vdW forces are generally expected to be important for molecular systems, nonlocal correlations are proving to be important for many other kinds of systems. For example, nonlocal correlations even play a significant role in the cohesion of covalently bound solids. ${ }^{35,50}$

The vdW-DF method aims to follow the same electrongas traditions as the LDA and the GGA, by building on a plasmon-pole description. . $^{3,11,12,44}$ Some details are presented in the Appendix.

The plasmon response is described by an inner functional that reflects LDA and gradient-corrected exchange. ${ }^{3}$ The nonlocal correlation can in turn be viewed as a counting of the plasmon zero-point energy shifts in the picture of Rapcewicz and Ashcroft. ${ }^{51}$ The total energy also includes an outer semilocal functional defined as LDA plus gradientcorrected exchange. This outer functional can be represented in terms of having a plasmon response. The vdW-DF versions, in general, all have deviations in the description of the exchange of the inner and outer functionals, i.e., they have a nonzero value for the cross-over term specified in Eq. (A12). Nevertheless, we seek to achieve a consistent treatment of exchange, that is, to use the same plasmon description for all functional components. Such consistency leads to an automatic conservation of the total $\mathrm{XC}$ hole. This goal guided our design of vdW-DF-xc. ${ }^{35}$

A fully consistent vdW-DF version, i.e., one with a good plasmon model, should be applicable for both dense and sparse matter, and thus have the potential for extending the dramatic success of constraint-based DFT. ${ }^{52}$ The new version, vdW-DF-cx, goes furthest in enforcing consistency between the inner and outer semilocal functionals. ${ }^{35}$ Given the emphasis on exchange consistency, the merits of a vdW-DF version boils down to exploring the quality of its plasmon-response description. ${ }^{35}$

\section{B. “Are we there yet?"}

Figure 1 presents an overview of the performance of different vdW-DF functionals based on (a) molecular dimers, [(b),(c)] layered materials, ${ }^{53}$ and (d) covalently bound solids. The overview suggests that the goal of designing a generalpurpose functional that works simultaneously for dense and sparse matter is a realistic one. This is true even if the versions differ in accuracy since the newer versions like vdW-DF2 and vdW-DF-cx perform better than older functionals like vdWDF1 and earlier special-purpose functionals. Nevertheless, the aim of a general-purpose functional should go beyond merely being able to describe different kinds of materials, like covalently bonded solids and dispersion-bound molecular dimers, it should also be able to describe the delicate balance between interactions in systems where different forces or ground-state configurations compete.

We have identified and addressed a number of systems where vdW forces compete with other interactions; with interactions spanning from strong ionic bonds to covalently bonded solids and physisorbed molecules within pores and at surfaces. We explore basic physical properties of these materials, such as lattice constants and adsorption energies, as well as the competition between the paraelectric and ferroelectric phases in $\mathrm{PbTiO}_{3}$ and the graphite and diamond phases of carbon. By examining these systems we show that vdW-DF-cx 

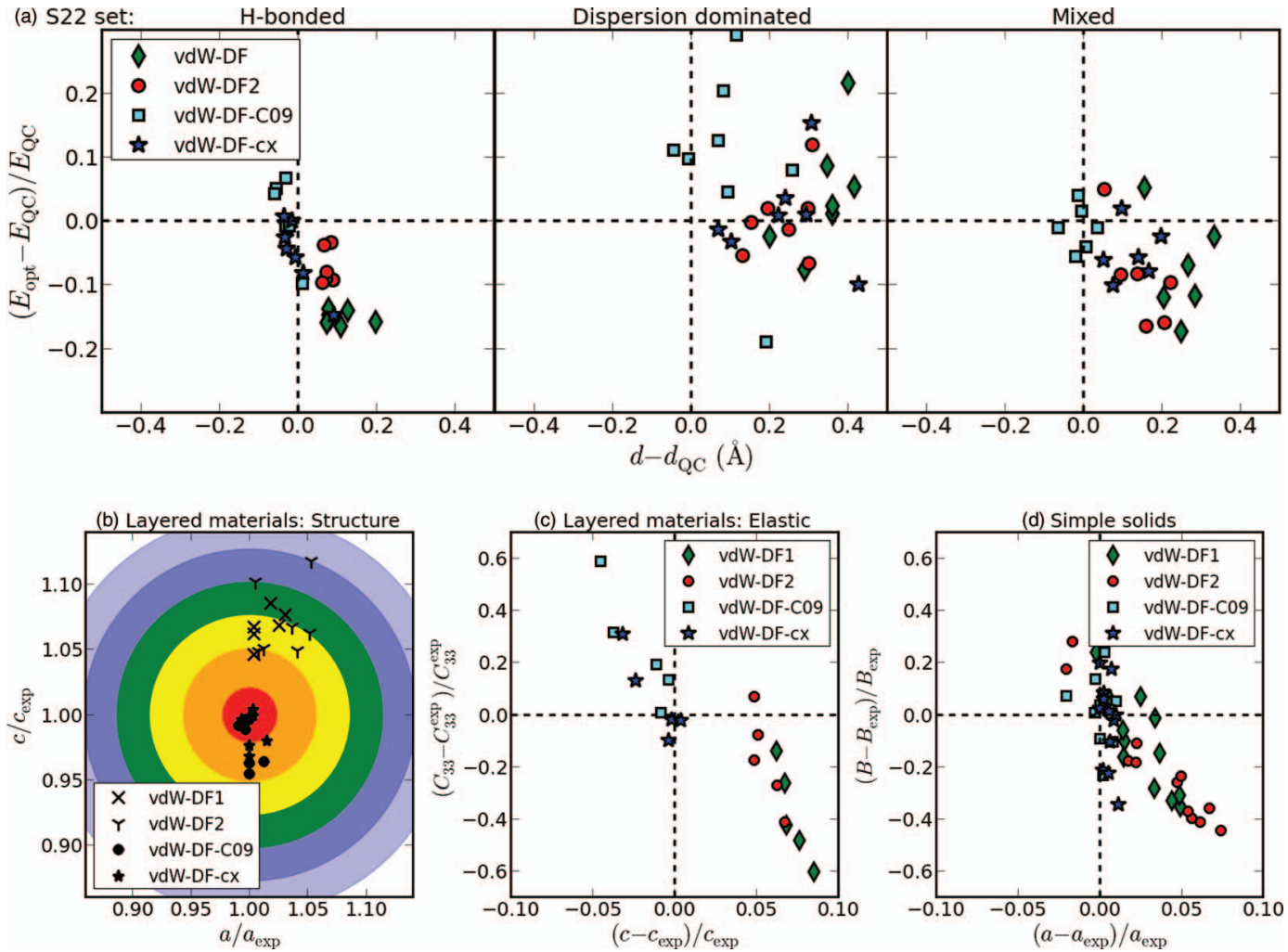

FIG. 1. Scatter-plots summarizing the performance of various vdW-DF versions $s^{3,5,33,35}$ for a set of tests: (a) The S22 benchmark, ${ }^{36}$ where values for binding energy $E_{\mathrm{opt}}$ and separations $d$ of sets of molecular dimers are compared with values from accurate quantum-chemistry calculations. (b) Comparison of calculated and experimental values ${ }^{119}$ for in- and out-of-plane lattice constants $a, c$ for a group of layered (or intercalated) compounds. (c) Comparison of calculated and experimental values of the out-of-plane lattice constants and the out-of plane $C_{33}$ elastic modulus. (d) Comparison of calculated and experimental values for the unit-cell lattice constant $a$ and bulk modulus $B$ for a group of simple solids (like $\mathrm{Al}, \mathrm{Ag}$, and $\mathrm{Au}$ ). Most calculated results are from Ref. 35, but we have also included results for potassium intercalation in graphite and for the variation of the $\mathrm{Bi}_{2} \mathrm{Te}_{3}$ layer binding energy with unit-cell size.

is indeed a general-purpose tool and as such holds significant promise as a unified functional for exploring sparse and dense matter alike.

\section{COMPUTATIONAL DETAILS}

For all systems, self-consistent vdW-DF calculations were performed using ultra-soft pseudopotentials (unless oth-

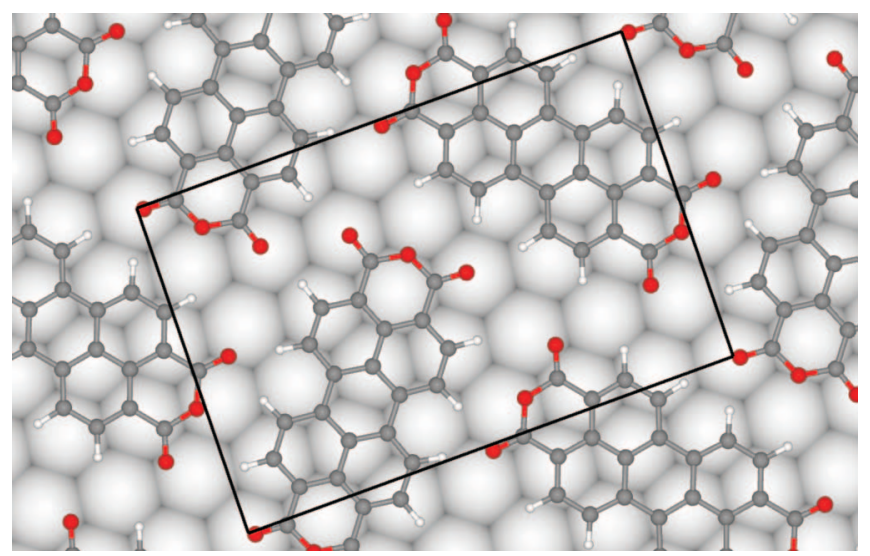

FIG. 2. The long-range ordered commensurate monolayer of PTCDA (perylene-3,4,9,10-tetracarboxylic-3,4,9,10-dianhydride) on $\mathrm{Ag}(111)$ taken from Ref. 54. Oxygen, carbon, hydrogen, and silver atoms are represented by red, grey, white, and light grey spheres, respectively. In the unit cell $\left(a=19.0 \AA, b=12.6 \AA, \gamma=89^{\circ}\right)$, there are two flat lying PTCDA molecules in a herringbone arrangement. erwise noted) as implemented in the QUANTUM ESPRESSO DFT package. We have implemented the vdW-DF-cx functional in QUANTUM ESPRESSO, which is now publicly available. The energy cutoff and k-point mesh were chosen to converge the binding energy to within $1 \mathrm{meV}$. All atoms were relaxed until the Hellmann-Feynman forces were less than $15 \mathrm{meV} / \AA$. We have used full stress relaxation ${ }^{55}$ of the unit cell unless otherwise noted.

For $\mathrm{PbTiO}_{3}$ a five-atom unit cell was employed. For the metal-organic-framework (MOF), we adopted the description in Ref. 56, using a limited wavevector sampling, as motivated by the large hexagonal unit cell $(a=25.881 \AA$ and $c=6.8789 \AA$ for Mg-MOF74; $a=25.887 \AA$ and $c=6.816 \AA$ for Zn-MOF74). These parameters reflect the experimental unit cell. The study of the adsorption of an organic molecule proceeded with a three-layer $\operatorname{Ag}(111)$ slab and 33 atoms per layer. The adsorption geometry is illustrated in the top panel of Fig. 2.

\section{RESULTS: TESTING NONLOCAL FUNCTIONALS WHEN FORCES COMPETE}

\section{A. A bulk-matter challenge: The ferroelectric response in $\mathrm{PbTiO}_{3}$}

$A B \mathrm{O}_{3}$ perovskites oxides (see Fig. 3, top panel) are exciting materials. A tremendous variety in their physical properties can be achieved as a result of the choice of 

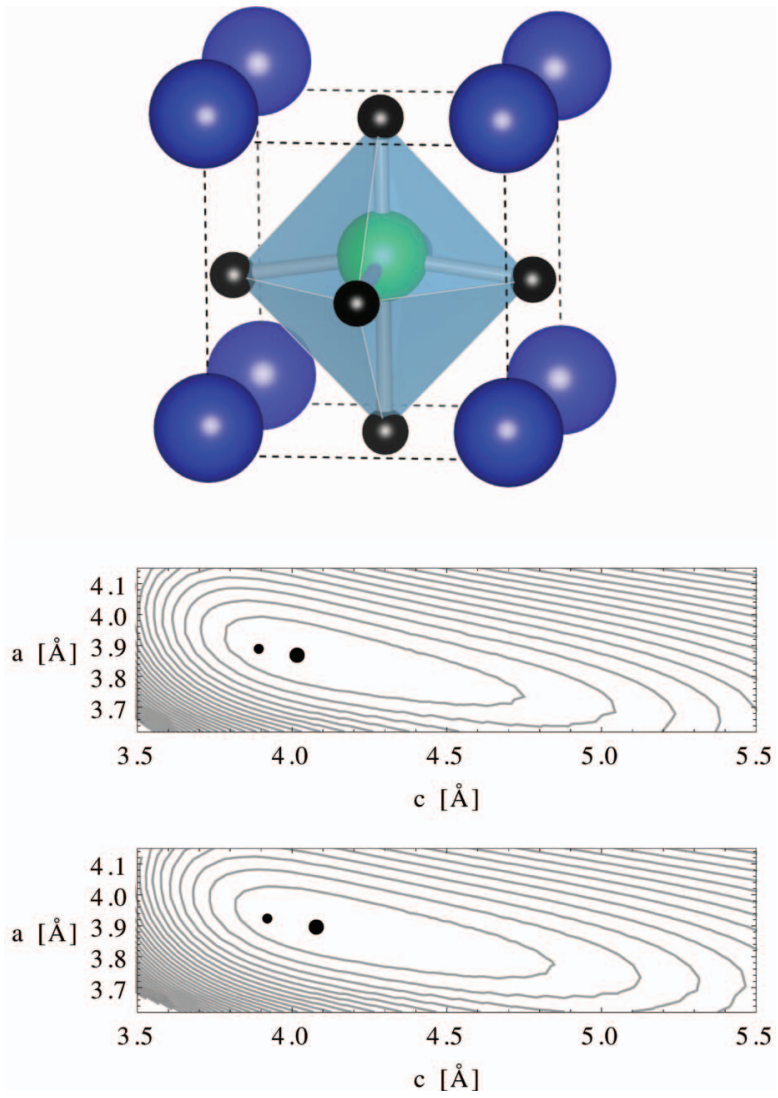

FIG. 3. Unit-cell atomic configuration (top panel) and potential energy surface (PES) of $\mathrm{PbTiO}_{3}$ (PTO) as evaluated in LDA (middle panel) and in vdW-DF-cx (lower panel). The internal energy (that is, the DFT total energy) variations are mapped by contours for the variation with unit-cell parameters " $c$ " and " $a$." The large (small) dot identifies the optimal geometry for the tetragonal (simple-cubic) phase. The contours shown are separated by $0.1 \mathrm{eV}$ relative to the DFT energy of the tetragonal state. Both LDA and vdW-DF-cx predict the experimentally correct polarization and both seem to have a good balance between exchange and correlation.

$A$ or $B$ site cations. They can be metals, band insulators, Mott insulators, superconductors, magnetic and ferroelectric with tunable coupling between these degrees of freedom. ${ }^{57-63}$ Ferroelectrics, and by extension piezoelectrics, have been of particular interest due to their current and potential technological importance. Ferroelectrics are characterized by a spontaneous polarization, which can be switched by an electric field, while piezoelectrics are a special subclass of ferroelectrics that exhibit a mechanical response that accompanies the change in polarization.

$\mathrm{PbTiO}_{3}$ (PTO) has been extensively studied both by theory and experiment due to its importance as an end member of many high-response piezoelectrics. PTO has a single phase transition from a cubic, paraelectric phase (the ideal perovskite structure) to a tetragonal, ferroelectric phase (with a large cla ratio - see mid and lower panels of Fig. 3) at a relatively high transition temperature, $T_{\mathrm{c}} \simeq 700 \mathrm{~K}$. PTO is a displacive ferroelectric in which the polarization is derived from the off-center displacements of the $A$ and $B$-site cations relative to their respective oxygen cages. The relatively large polarization of PTO is related to both the presence of a ferroelectrically active $A$-site cation and the partially covalent bonding of the $\mathrm{Ti}$ ions, which results in anomalously large Born effective charges. ${ }^{64}$

Typically, DFT calculations of bulk oxide ferroelectrics have employed LDA for exchange and correlation. This is largely due to the fact that GGAs overestimation of the lattice constant results in it severely failing to describe the local structure of ferroelectric oxides. LDA only modestly underestimates lattice constants and thus performs reasonably well. ${ }^{65,66}$ In any event, this description of the structure and energetics of the relevant phase is particularly problematic when attempting to predict $T_{\mathrm{c}}$ as they are closely related to both the polarization and the energy difference between the paraelectric and ferroelectric phases. Naturally, LDA consistently underestimates $T_{\mathrm{c}}$. Negative pressure simulations ${ }^{61,67,68}$ and new functionals ${ }^{69-71}$ have been invoked to correct for these errors in DFT. Indeed, we find that PBEsol $^{71}$ (unlike $\mathrm{PBE}^{28}$ ) provides an accurate description of the PTO atomic displacements and polarization in the groundstate.

To fully characterize PTO we must be able to both describe the ground-state structure and the atomic displacements as the material makes the transition from the ground-state ferroelectric phase to the high-temperature paraelectric phase. Even though bulk-oxide ferroelectric forces are not typical sparse matter systems, we deem that they are good test systems for vdW-DF-cx. Due to strong competition between long range Coulombic interactions and local covalent interactions, small differences in the nonlocal correlation energy could possibly tip the balance between the high-temperature cubic phase and the groundstate tetragonal phase.

Table I details our comparison of the performance of different functionals in terms of structure, thermal stability, and the internal displacements of atoms. vdW-DF1 and vdW-DF2 significantly overestimate the $c$ lattice constant and predict dramatic internal distortions. vdW-DF2 also predicts a very large energy difference between the phases. vdW-DF-cx and vdW-DF1-C09 have the best performance in terms of displacements and energetic difference of the phases.

The ground-state structure has characteristic atom separations of $2.25 \AA$ indicating that nonlocal correlations can be important. Indeed, we find that for all considered vdW-DF versions the energy difference between the $E_{c}^{\mathrm{nl}}$ contributions to the energy of the two phases is larger than the total change in internal energy.

\section{B. Small-molecular absorption: Weak chemisorption in a metal-organic framework}

With the development of vdW-DF and other sparse-mater methods, DFT has become a key tool for the screening of potential MOF candidates for technologically important applications such as hydrogen storage and gas sequestration for carbon capture. ${ }^{72-74}$ Describing the adsorption within MOFs is however challenging because of the presence of metal ions which introduce an electrostatic component to the binding as well as covalent effects. Due to these competing interactions, MOFs present an ideal testing ground for methods aiming to describe the crossover from weak, to moderate, and strong chemisorption. 
TABLE I. Structural and electronic properties of PTO. $a$ and $c$ denote the equilibrium in-plane and out-ofplane lattice parameters. $\mathrm{P}_{z}, \delta E_{\text {phase }}$, and $\delta E_{\text {phase }}^{\mathrm{c}, \mathrm{nl}}$ are the magnitude of the spontaneous polarization, the energy difference between the cubic and tetragonal phases and the nonlocal correlation component of the total energy, respectively. The polarization vector $P$ is aligned with the $c$ axis. The table also lists atomic positions $\xi_{z}$ (the crystal-coordinate vectors projected on the polarization direction) for the tetragonal phase, with the unit-cell offset as defined in Ref. 57.

\begin{tabular}{lcccccc}
\hline \hline & vdW-DF1 & vdW-DF2 & vdW-DF-C09 & vdW-DF-cx & Expt. & LDA \\
\hline$a(\AA)$ & 3.8741 & 3.8893 & 3.8927 & 3.8955 & $3.9040^{\mathrm{a}}$ & 3.8663 \\
$c(\AA)$ & 4.8152 & 4.8681 & 4.0518 & 4.0788 & $4.1575^{\mathrm{a}}$ & 4.0190 \\
$\mathrm{P}_{z}\left(\mathrm{C} / \mathrm{m}^{2}\right)$ & 1.22 & 1.19 & 0.72 & 0.75 & $0.75^{\mathrm{b}}$ & 0.74 \\
$\delta E_{\text {phase }}(\mathrm{eV})$ & -0.002 & -0.265 & -0.047 & -0.055 & $\ldots$ & -0.053 \\
$\delta E_{\text {phase }}^{\mathrm{c} \text {,nl }}(\mathrm{eV})$ & 0.018 & 0.298 & 0.080 & 0.092 & $\ldots$ & \\
$\xi_{z}(\mathrm{~Pb})$ & 0.16613 & 0.16328 & 0.09199 & 0.09676 & $0.1206(6)^{\mathrm{a}}$ & 0.09709 \\
$\xi_{z}(\mathrm{Ti})$ & 0.60949 & 0.60919 & 0.56489 & 0.56808 & $0.5773(4)^{\mathrm{a}}$ & 0.56592 \\
$\xi_{z}\left(\mathrm{O}_{I}\right)$ & -0.02737 & -0.02864 & 0.00958 & 0.00853 & $-0.0077(9)^{\mathrm{a}}$ & 0.01265 \\
$\xi_{z}\left(\mathrm{O}_{I I / I I I}\right)$ & 0.5 & 0.5 & 0.5 & 0.5 & $0.5^{\mathrm{a}}$ & 0.5 \\
\hline \hline
\end{tabular}

${ }^{\mathrm{a}}$ Reference 57.

${ }^{\mathrm{b}}$ Reference 58 .

Figure 4 shows a calculated binding geometry of $\mathrm{CO}_{2}$ in Mg-MOF74. Similar to previous studies, ${ }^{56,75}$ our calculations show that the $\mathrm{CO}_{2}$ adsorption in Mg-MOF74 is governed by $\mathrm{vdW}$ forces. However, replacing $\mathrm{Mg}$ in the same framework with Al can lead to strong chemisorption, which can even significantly deform the adsorbed molecule. ${ }^{75}$ In this study, we limit ourselves to assessing how vdW-DF-cx performs for the adsorption of $\mathrm{H}_{2}$ and $\mathrm{CO}_{2}$ within $\mathrm{Zn-MOF74}$ and Mg-MOF74.

Table II shows a comparison of the performance of different functionals. vdW-DF-cx like vdW-DF1 predicts similar binding energies, which are significantly overestimated for $\mathrm{H}_{2}$ adsorption, but in good agreement with experiments for $\mathrm{CO}_{2}$

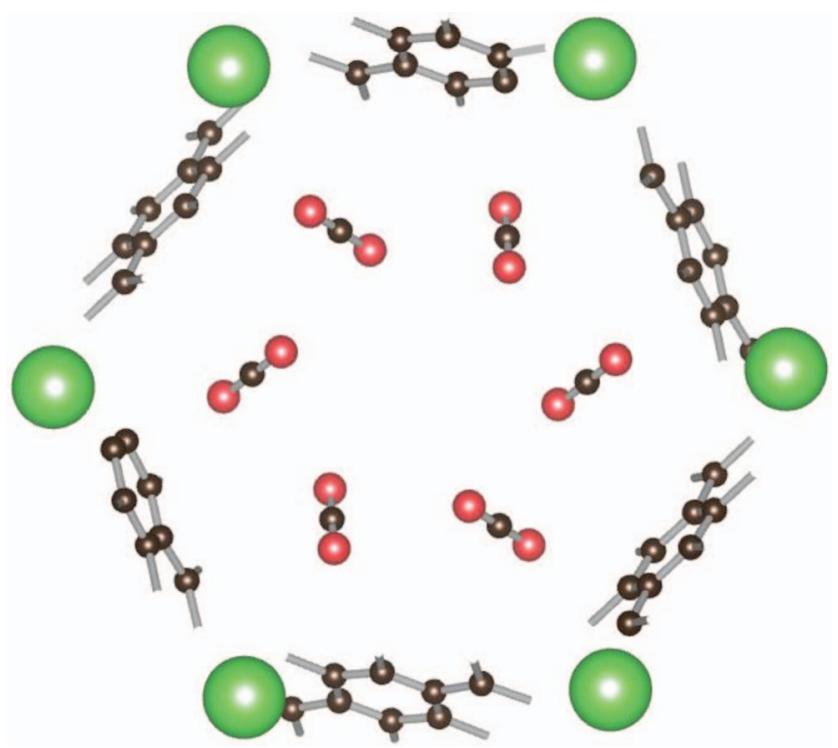

FIG. 4. Final relaxed structure of the $\mathrm{CO}_{2}$ adsorption in the $\mathrm{Mg}-\mathrm{MOF} 74$ structure as computed with vdW-DF-cx. For all vdW-DF versions, the MOF74 unit cell was kept fixed at the experimental value while all atoms were allowed to relax. For the $\mathrm{H}_{2}$ incorporation we found no discernable difference between adsorbing a single or the full load of six absorbates. In the case of $\mathrm{CO}_{2}$, the absorption energy was found to be $20 \mathrm{meV}$ deeper with 6 instead of a single molecule at the $\mathrm{Mg}$ site. Table II summarizes the details of our absorption study and compares with experimental studies and results of other methods where available. adsorption. vdW-DF-cx predicts slightly shorter separations than vdW-DF2, yielding a slightly better agreement with the experimental separation for $\mathrm{CO}_{2}$ in $\mathrm{Mg}-\mathrm{MOF} 74$, the only system where this quantity is experimentally available. Overall vdW-DF2 is the best method for the considered systems. Like in earlier MOF studies, ${ }^{76}$ the ability of vdW-DF functionals to be applicable on both small-molecule and expanded-lattice scales should be noted.

\section{Graphite-intercalated system}

Graphite intercalation is relevant for battery operation since the alkali uptake can be electrochemically controlled. The resulting staging of the graphite matrix produces a dramatic increase in the graphene sheet-to-sheet separation in concert with a charge transfer to the sheets. The charge transfer is known to buckle the graphene sheets.

This industrial relevance motivated an early vdW-DF1 study on the formation energy and response of $\mathrm{C}_{8} \mathrm{~K} .{ }^{48} \mathrm{How}-$ ever, despite the charge transfer in this system, vdW-DF1 still overestimated the out-of-plane lattice constant by $0.2 \AA$. It is therefore interesting to apply the new vdW-DF-cx functional to this problem. Our new vdW-DF1 calculations also supersede the old ones because the new calculations are selfconsistent and allow for atomic relaxations, capturing the charge-induced sheet buckling.

Table III presents our results for $\mathrm{C}_{8} \mathrm{~K}$ and graphite. Overall we find that the description of the potassium intercalation is improved by the self-consistent relaxation. We also find that vdW-DF-cx provides the most accurate description of the $\mathrm{C}_{8} \mathrm{~K}$ structure and behavior among the vdW-DF versions investigated.

\section{The graphite/diamond phase transition}

Graphite is the ground state among carbon allotropesbut only just so, as the binding energy difference to diamond is small. It takes a large pressure to produce diamonds directly from graphite but that is due to a large kinetic barrier. From analysis of the high temperature phase behavior and other thermal behaviors it is possible to extract an estimate of 
TABLE II. Adsorption energies $E_{\text {ads }}$ (in $\mathrm{eV}$ ) and distance $d$ (in $\AA$ ) from the closest atom of the adsorbed molecule to the metal site for $\mathrm{H}_{2}$ and $\mathrm{CO}_{2}$ adsorbed in Mg-MOF74 and Zn-MOF74. For comparison, references to results from LDA, GGA, and PBE-D studies are also included where available.

\begin{tabular}{lcrrrcccc}
\hline \hline & & vdW-DF1 & vdW-DF2 & vdW-DF-cx & Expt. & LDA & GGA & PBE-D \\
\hline $\mathrm{H}_{2}$ in Zn-MOF74 & $d$ & 3.223 & 2.848 & 2.736 & $\ldots$ & $\ldots$ & $2.83^{\mathrm{a}}$ & $\ldots$ \\
& $E_{\text {ads }}$ & -0.135 & -0.119 & -0.137 & $-0.091^{\mathrm{a}}$ & $-0.228^{\mathrm{a}}$ & $-0.046^{\mathrm{a}}$ & $\ldots$ \\
$\mathrm{H}_{2}$ in Mg-MOF74 & $d$ & 2.729 & 2.526 & 2.535 & $\ldots$ & $\ldots$ & $2.54^{\mathrm{a}}$ & $\ldots$ \\
& $E_{\text {ads }}$ & -0.163 & -0.155 & -0.171 & $-0.105^{\mathrm{a}}$ & $-0.257^{\mathrm{a}}$ & $-0.062^{\mathrm{a}}$ & $\ldots$ \\
$\mathrm{CO}_{2}$ in Zn-MOF74 & $d$ & 3.007 & 2.835 & 2.752 & $\ldots$ & $\ldots$ & $\ldots$ & $2.71^{\mathrm{b}}$ \\
& $E_{\text {ads }}$ & -0.381 & -0.313 & -0.371 & $\ldots$ & $\ldots$ & $\ldots$ & $-0.309^{\mathrm{b}}$ \\
$\mathrm{CO}_{2}$ in Mg-MOF74 & $d$ & 2.401 & 2.341 & 2.325 & $2.283^{\mathrm{c}}$ & $\ldots$ & $\ldots$ & $2.45^{\mathrm{b}}$ \\
& $E_{\text {ads }}$ & -0.520 & -0.473 & -0.520 & $-0.487^{\mathrm{b}}$ & $\ldots$ & $\ldots$ & $-0.429^{\mathrm{b}}$ \\
\hline \hline
\end{tabular}

${ }^{a}$ Values taken from Ref. 77.

${ }^{\mathrm{b}}$ Values taken from Ref. 78.

${ }^{\mathrm{c}}$ Values taken from Ref. 79.

what would be the equilibrium phase difference in enthalpy or equivalently an estimate of what pressure is required to induce a phase transition (ignoring the kinetic barrier). In this procedure, the phase separation is estimated to have approximately a $0.7 \mathrm{GPa}$ equilibrium transformation pressure. ${ }^{81,82}$

Since graphite is a partly vdW bonded system with $\mathrm{sp}^{2}$ bonds between the atoms of the graphene sheet, while diamond is purely $\mathrm{sp}^{3}$ bonded, it becomes interesting to test the accuracy of a set of nonempirical descriptions of this transition.

Our results are summarized in Table III. Interestingly, we find that while LDA does provide a finite binding between the layers, it also produces an incorrect ordering of the enthalpy of graphite and diamond phases. The set of nonlocal functionals all produce a binding of the graphite layers, although vdW-DF1 and vdW-DF2 have a very high value for the predicted differences in the phase enthalpy.

Overall we observe that vdW-DF-cx produces a prediction for the energy ordering that is closest to the carbonallotrope behavior. However, we note we have not at this stage added lattice zero-point energy effects.

\section{E. Organic-molecule adsorption on $\mathrm{Ag}(111)$}

The adsorption of organic molecules on coinage metal surfaces is a problem where different sparse matter methods can yield widely varying results, ranging from covalent binding with short molecule-to-surface separations to a purely dispersion-bounded description as typically predicted with vdW-DF1. ${ }^{88-91}$ These systems are therefore particularly challenging ones.

TABLE III. Structure and binding of graphite intercalation and graphite. Unit-cell parameters (in-plane lattice constant $a$ and average separation $d_{\mathrm{C}-\mathrm{C}}$ between carbon sheets) are given in $\AA$. The graphite interlayer binding energy $\Delta E_{\text {bind }}^{\text {lay }}$ is given in meV per graphene-sheet atom. The graphite $\mathrm{AA}$-vs- $\mathrm{AB}$ stacking fault energy $\Delta E_{\mathrm{SF}}^{\mathrm{AA}}$ and the graphite-versus-diamond internal-energy difference $\Delta E_{\text {phase }}^{\mathrm{G} / 3 \mathrm{C}}$ are given in meV per graphite unit cell. The corresponding phase transition pressure $p$ is estimated by simply dividing by the vdW-DF-cx estimate for the graphite-to-diamond volume change per atom, $\delta V_{C} \approx 3 \AA^{3}$. The $\mathrm{C}_{8} \mathrm{~K}$ formation energy $\Delta E_{\text {form }}^{\mathrm{Katom}}$ is given in $\mathrm{eV}$ per $\mathrm{C}_{8} \mathrm{~K}$ formula unit and relative to the internal energy of graphite and of free potassium atoms. In the LDA calculation (but not for any vdW-DF studies) we include the effect of a small spin-polarization energy ( $26 \mathrm{meV})$ for these potassium atoms. As indicated by a pair of "NA" entries, the PBE does not give any meaningful account of graphite binding (it is nominally computed as $1.2 \mathrm{meV}$ per graphene-sheet atom) and there consequently exists no PBE account of the $\mathrm{C}_{8} \mathrm{~K}$ formation energy either. Because the enthalpy difference between phases is so small in vdW-DF-cx we have also carried out a check using normconserving pseudopotentials, with results given in square brackets.

\begin{tabular}{|c|c|c|c|c|c|c|c|}
\hline & & vdW-DF1 & vdW-DF2 & vdW-DF-cx & Expt. & LDA & GGA \\
\hline \multirow[t]{6}{*}{ Graphite } & $a$ & 2.473 & 2.478 & $2.466[2.467]$ & $2.459^{\mathrm{a}}$ & 2.466 & 2.467 \\
\hline & $d_{\mathrm{C}-\mathrm{C}}$ & 3.581 & 3.517 & $3.275[3.284]$ & $3.336^{\mathrm{a}}$ & 3.325 & 4.063 \\
\hline & $\Delta E_{\text {bind }}^{\text {lay }}$ & 55 & 53 & $66^{67}$ & $52^{\mathrm{b}}$ & 25 & NA \\
\hline & $\Delta E_{\mathrm{SF}}^{\mathrm{AA}}$ & 19 & 24 & $47^{47}$ & & 39 & 1.3 \\
\hline & $\Delta E_{\mathrm{phase}}^{\mathrm{G} / 3 \mathrm{C}}$ & 790 & 1129 & $7^{30}$ & & -100 & NA \\
\hline & $\Delta p_{\text {phase }}^{\mathrm{G} / 3 \mathrm{C}}$ & 11 & 15 & $0.09[0.4]$ & $0.7^{\mathrm{c}, \mathrm{d}} / 1.4^{\mathrm{e}}$ & -1.4 & NA \\
\hline \multirow[t]{3}{*}{$\mathrm{C}_{8} \mathrm{~K}$} & $a$ & 2.494 & 2.497 & 2.487 & $2.480^{\mathrm{f}}$ & 2.485 & 2.490 \\
\hline & $d_{\mathrm{C}-\mathrm{C}}$ & 5.44 & 5.43 & 5.22 & $5.35^{\mathrm{g}}$ & 5.17 & 5.37 \\
\hline & $\Delta E_{\text {form }}^{\text {Katom }}$ & 1.00 & 0.93 & 1.29 & $1.24^{\mathrm{h}}$ & 1.67 & NA \\
\hline
\end{tabular}

${ }^{\mathrm{a}}$ Reference 80.

${ }^{\mathrm{b}}$ Reference 84 .

${ }^{\mathrm{c}}$ Reference 81

${ }^{\mathrm{d}}$ Reference 82.

${ }^{\mathrm{e}}$ Reference 83

${ }^{\mathrm{f}}$ Reference 85 .

${ }^{\mathrm{g}}$ Reference 86

${ }^{\mathrm{h}}$ Reference 87. 
A prototypical model system is the 3,4,9,10-perylenetetracarboxylic-dianhydride (PTCDA) molecule on $\operatorname{Ag}(111)$, which forms a long-range ordered commensurate monolayer and has been studied extensively both experimentally and theoretically. ${ }^{54,92-98}$ Results for the lateral adsorption structure (Figure 2) and vertical adsorption bond lengths are available. The system therefore represents a valuable benchmarking system.

For a straightforward comparison with previous work by Ruiz and co-workers, ${ }^{98}$ we first assume that both the surface and the molecules are flat and rigid, before doing a full geometry optimization. The Ag substrate structure is kept frozen at the atomic configuration obtained from experimental lattice parameters of bulk Ag. The potential energy curves (PECs) of the PTCDA monolayer as a function of the vertical separation of the layer are compared in Fig. 5, along with the results from Ref. 98. The fully relaxed results, including both the relaxation of the interface and the intermolecular interaction energy between PTCDA molecules within the layer, are indicated by cross-marks in Fig. 5 and will be discussed further below.

The vdW-DF-cx performs similarly to $\mathrm{PBE}+\mathrm{vdW}$ surf. Both show good agreement with the experimental binding separation, while vdW-DF1 and vdW-DF2 overestimate it. A comparison of the adsorption energy is, however, not straightforward. Determining the adsorption energy of PTCDA on $\operatorname{Ag}(111)$ is difficult and we only have a rough estimate for an experimental value. Standard temperature-programmed desorption (TPD) is inapplicable because the molecule fragments before desorption. ${ }^{99}$ Tkatchenko and co-workers ${ }^{97}$ estimate the binding energy to be $2.4 \mathrm{eV}$ from a value that is twice that of the desorption energy of a smaller, but similar molecule, naphthalenetetracarboxylic dianhydride (NTCDA), which is roughly half the size of PTCDA. ${ }^{100}$

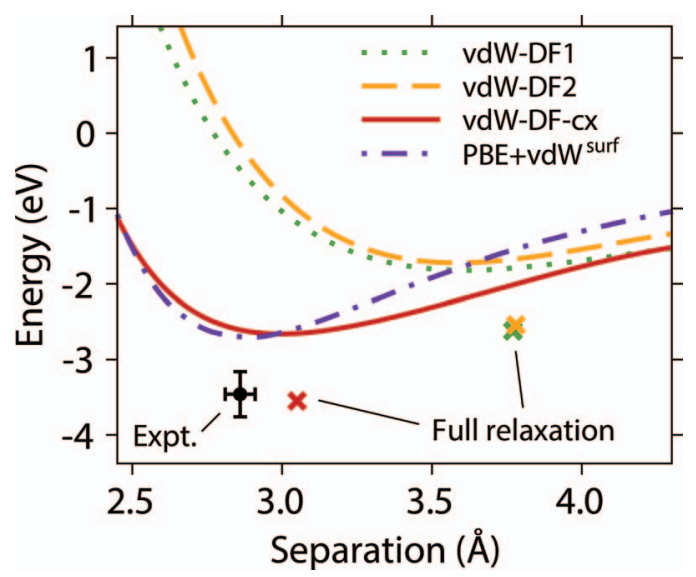

FIG. 5. Potential energy curves (PECs) for an epitaxial monolayer of PTCDA on a $\operatorname{Ag}(111)$ substrate as a function of the vertical separation calculated using three vdW-DF versions and $\mathrm{PBE}+\mathrm{vdW}^{\text {surf }}$, assuming flat and rigid molecules and surface. The cross marks are the adsorption energies obtained by performing a full relaxation of the interface, i.e., both the molecule and the first atomic layer of the slab, and by including intermolecular interactions. The experimental values for the binding energy and separation distance are shown as a black dot with error-bars. The PECs are calculated in the same three-layer slab representation that was used in an earlier empirical pair-potential plus image-plane correction study. ${ }^{98}$
We argue, however, that the binding energy may be significantly larger for the following reasons: First, the desorption energy of NTCDA $(1.16 \mathrm{eV})$ was obtained ${ }^{100}$ for a loosely packed monolayer at low coverage, in which intermolecular hydrogen bonds are weak or missing. According to our calculation using vdW-DF-cx, intermolecular interactions contribute to the desorption energy by $0.46 \mathrm{eV}$ per molecule. Further, Fichthorn and co-workers showed ${ }^{101}$ that for large molecules the pre-exponential factor in the Redhead formula for TPD should be several orders of magnitude larger than a typical value for smaller ones due to the large entropy associated with many local minima. For example, the use of a typical pre-exponential factor underestimates the desorption energy of dodecan $\left(\mathrm{C}_{12} \mathrm{H}_{26}\right)$ on $\mathrm{Au}(111)^{101}$ by $25 \%$. Applying the same ratio of underestimation and by including an intermolecular interaction energy correction, we estimate the binding energy to be $3.46 \mathrm{eV}(2.4 \times 1.25+0.46 \mathrm{eV})$. The corresponding adsorption energy predicted by vdW-DF-cx falls within this experimental estimate, which presumably will be the case for PBE+vdW ${ }^{\text {surf }}$. However, vdW-DF1 and vdWDF2 predict smaller energies.

In order to quantify the degree of structural changes of the molecule and the surface separately, we first optimized the structure of the molecule while keeping the flat surface intact. Next, a full relaxation of the interface was performed; including the first atomic layer of the surface and keeping the bottom two atomic layers fixed.

The energy gained from molecular relaxations, $0.36 \mathrm{eV}$ per molecule, is much larger than that gained via the surface relaxation, $0.07 \mathrm{eV}$ per molecule. After full relaxation of the interface and including the intermolecular interaction energy, the calculated binding energy is $3.55 \mathrm{eV} .{ }^{102}$ We obtained a similar amount of stabilization with vdW-DF1 and vdW-DF2.

In this system, it is the nonlocal correlation and a weak chemisorption component that attract the molecules to the $\mathrm{Ag}$ substrate. Semilocal functionals like PBE cannot accurately describe this adsorption problem; the van der Waals forces are needed to pull the molecules closer to the surface.

Additionally, for some systems, we need a reliable account of the bonding within the surface in order to describe the adsorption of molecules. ${ }^{34,50}$ This is important for capturing the adsorption-induced surface relaxations, though this effect is less important for the adsorption case studied here. Nevertheless, the vdW-DF-cx version can reliably describe both the molecular adhesion and can, for example, accurately describe the $\mathrm{Ag}$ bulk structure. It is worth noting that the optB86b exchange functional has also been demonstrated to show superior performance for the adsorption of organic molecules ${ }^{103-105}$ to coinage metal surfaces as well as for accurately describing the structure of many bulk metals. ${ }^{50}$ As such, we believe that it may also be capable of describing a wide range of length scales and interactions.

\section{DISCUSSION OF vDW-DF VERSIONS}

We here discuss the nature of the different nonempirical vdW-DFs developed in our collaboration. This serves to put vdW-DF-cx in the context of these earlier developments. 


\section{A. vdW-DF1}

$v d W-D F 1^{3}$ uses revPBE ${ }^{30}$ as the exchange component of the outer functional $E_{x c}^{\mathrm{sl}}[n]$ that describes most of the exchange part of the total energy. This choice was made because revPBE is a well tested functional that in practice ensures that no unphysical binding arises from exchange alone as, for example, in the case of the benzene dimer. ${ }^{3,13,15,106,107}$

The exchange functional is usually described in terms of the reduced density gradient $s=|\nabla n| / 2 k_{\mathrm{F}} n$ and the exchange enhancement factor $F_{\mathrm{x}}(s)$. The enhancement factor of revPBE is similar to that of PBE, in particular they agree for small $s$ values. As an alternative to the constraint-based derivation, the PBE functional can be justified by its similarity to the numerical GGA. ${ }^{29}$ This GGA form is based on imposing a cutoff of the gradient expansion approximation (GEA) for the $\mathrm{XC}$ hole to preserve its unit charge, a procedure that among other things generalizes the analysis in the construction of the PW86 exchange. ${ }^{27,32} \mathrm{vdW}-\mathrm{DF} 1$ therefore benefits from some of the insights that underpin the construction of the PBE. However, we stress that this design guide is implemented only for the exchange content in $E_{x c}^{\mathrm{sl}}$, which ignores residual exchange in $E_{c}^{\mathrm{nl}}$. We also note that a modulation factor of the GEA hole was used so that its exchange form differs from a direct implementation of exchange-hole conservation ${ }^{32}$ at small $s$ values; the large-s enhancement factor of the numerical GGA was ignored altogether in specifying the PBE exchange.

Nevertheless, vdW-DF1 successfully describes the binding in many dispersion bound (sparse matter) systems. ${ }^{7,88}$ For instance, it does quite well for computing the energetics of layered systems, ${ }^{35,48,108}$ as illustrated by the graphite case in Table III. The fact that revPBE is quite repulsive, however, causes vdW-DF1 to systematically produce too large separations in molecular systems and layered materials. $32,35,50,109$ This is illustrated respectively by the S22 data shown in Fig. 1(a) and for the layered systems in Fig. 1(b). In addition, the lattice constants of many inorganic solids are overestimated, as seen in Fig. 1(d). The latter can be an issue when modelling the adsorption of molecules, because it can be important for permitting the near-surface atoms to relax. ${ }^{50}$ The $\mathrm{PBE}^{28}$ functional also tends to overestimate lattice constants, a feature that has been linked to the fact that its enhancement factor differs from that obtained from an analysis of the manybody diagrams for the weakly perturbed HEG. ${ }^{21,24,25,71,110}$ It is plausible that the lattice-constant overestimation is exacerbated in vdW-DF1 because it does not retain a LDA-type description of linear response and has a different balance between gradient corrections to exchange and correlation. ${ }^{71,110}$

\section{B. vdW-DF2}

vdW-DF2 employs an exchange-enhancement description for the inner functional $E_{x c}^{0, i}[n]$ obtained from the formal results of Schwinger and of Elliott and Burke. ${ }^{11,112}$ This is in contrast to using the many-body results of the HEG to describe the plasmon response, as was done in the design of the vdW-DF1. The approach is demonstrated to be accurate for atoms ${ }^{113}$ and can be shown ${ }^{112}$ to essentially have the character of the Becke- 88 functional ${ }^{114}$ for small values of the scaled gradient. This is one factor indicating good performance for molecules.

vdW-DF2 also updates the choice of exchange component of the outer functional to the revised PW86. ${ }^{32}$ The enhancement factor of this functional arises from enforcing a hard cutoff on the exchange-hole of GEA. ${ }^{27}$ Since it has been shown that this exchange choice agrees well with that of a Hartree-Fock description of exchange effects between molecules, ${ }^{32,115}$ one can expect vdW-DF2 to have high accuracy and good transferability for small-molecular systems. ${ }^{5}$

vdW-DF2 indeed performs well for many types of systems, including dimers ${ }^{5}$ and the adsorption of small molecules. ${ }^{91,109,116}$ It significantly improves upon the account of vdW-DF1. These trends are captured in the scatter plots for the S22 data set in Fig. 1(a), as well as for the adsorption in MOF74 listed in Table II. However, vdW-DF2 only slightly improves interlayer separations of layered compounds relative to vdW-DF1, ${ }^{108}$ as shown in Fig. 1(b). Further, the adsorption energy for bigger molecules can be somewhat underestimated, ${ }^{45}$ as seen in Fig. 5 and the value of lattice constants of solids usually worsens, Fig. 1(d). ${ }^{35,50}$ This lack of improvement for solids and larger molecular systems is expected since neither the inner nor the outer functional has an exchange-enhancement factor that is consistent with the results of a many-body physics analysis for a weakly perturbed electron gas. ${ }^{21-29,71,110}$

We note that vdW-DF2 has fair consistency between the inner exchange parameterizing the plasmons (the plasmons that set the determination of nonlocal correlation $E_{c}^{\mathrm{nl}}$ ) and the exchange part of the outer functional $E_{x c}^{\mathrm{sl}}$ (the semilocal part of the full XC energy) at least at small to moderate values of the reduced gradient $s$. This fair consistency was not used to motivate the vdW-DF2 design but follows from the fact that it systematically implements GGA-type descriptions that are excellent for (small-)molecule-type problems.

\section{C. vdW-DF-cx}

$v d W-D F-c x^{35}$ effectively implements the conditions necessary for consistency between the inner and outer exchange. Starting with the vdW-DF1 plasmon-pole description of the inner-functional and hence nonlocal correlations, Ref. 35 demonstrates that the range of $s$-values that contributes to $E_{c}^{\mathrm{nl}}[n]$ is limited to $s<2-3$ for most material properties of interest. It is therefore sufficient to demand consistency of the inner and outer functional descriptions in this limited regime. At the same time, the $E_{c}^{\mathrm{nl}}$ analysis of the relevant contributing $s$ values also implies that for $s>2-3$ one can proceed with a traditional (numerical-GGA) analysis, ${ }^{27,32}$ to specify an exchange enhancement factor for the large-s regime. Overall we arrive at an outer-functional exchange specification, termed LV-rPW86, that is designed exclusively with the purpose of working with the vdW-DF1 description of nonlocal correlations. The result is a nonempirical vdW-DF version, vdWDF-cx ${ }^{35}$ that effectively ensures hole conservation for most materials problems; the only exception being $\mathrm{vdW}$ binding of noble gas atoms and small molecules, for reasons discussed in Ref. 35. 
Since the plasmons giving rise to the nonlocal correlation of vdW-DF1 are described by a near-HEG behavior, ${ }^{35} \mathrm{vdW}-$ DF-cx has an exchange component which is not far from that of previous exchange functionals used with vdW-DF1, like $\mathrm{C} 09 \mathrm{x}^{33}$ and optB86b. ${ }^{50}$ Both of these functionals resemble the design logic of the PBEsol functional ${ }^{71}$ for small-to-medium $s$ values (but with different large-s tails) and have significant improvements in lattice-constants over vdW-DF1. Albeit, neither $\mathrm{C} 09 \mathrm{x}$ nor optB86b were designed to minimize the value of the crossover term expressed in Eq. (A12), and thus cannot leverage the conservation of the total $\mathrm{XC}$ hole that is implied (for a plasmon-pole description) in Eq. (A5).

Given the strong connection with PBEsol it is not surprising that the new vdW-DF-cx functional improves upon lattice constants for both bulk and layered vdW systems, as shown in the lower panels of Fig. $1 .{ }^{35}$ For systems involving the smallest of molecules, like $\mathrm{H}_{2}$ within MOF74, vdW-DF2 is more appropriate. Nevertheless, vdW-DF-cx comes out in overall slightly better than vdW-DF2 for the S22 benchmark set of molecular dimers. Systems characterized by competing interactions are often those where vdW-DF-cx does particularly well. In addition to the importance of using a consistent exchange account, this trend can be related to the fact that these systems are characterized by shorter separations than the purely vdW bonded ones. These shorter separations make the near-HEG description, that both the correlation and exchange of vdW-DF-cx rely on, more appropriate. It is encouraging that vdW-DF-cx improves the description of binding energies in systems that range from bulk systems, to layered compounds and molecular systems, as well as to systems characterized by competing interactions.

\section{CONCLUSION}

Good tools are valuable for the theoretical description and exploration of general matter; i.e., systems that are comprised of regions of both dense and sparse electron concentrations. For ground-state properties DFT has been available for almost half a century. During the last decade its application to sparse (and hence general) matter has been significantly improved. The variety of relevant systems is enormous, far beyond those indicated by the applications in this paper: the ferroelectric response in $\mathrm{PbTiO}_{3}$, the adsorption of small molecules in MOFs, the phase transition between graphite and diamond, and the adsorption of an organic-molecule on the $\mathrm{Ag}(111)$ surface. Nevertheless, the selected examples are sufficient enough to represent the variety of possible materials challenges.

The nonempirical vdW-DF method, the tool of this study, is characterized by high ambitions of simplicity and physicality. The applications are performed with four vdW-DF functionals, vdW-DF1, vdW-DF2, vdW-DF using C09 exchange, and the very recent vdW-DF-cx. ${ }^{35}$ In a revitalization of the development of the vdW-DF method we have particularly noticed that it benefits from extensive studies of the almost homogeneous electron gas, including those leading up to the GGAs. This is the electron-gas tradition of a plasmonpole description. ${ }^{3,19,23,41-43}$ Our recent analysis ${ }^{35}$ shows that this plasmon description should be used within vdW-DF to give a good description of both exchange and correlation effects.

The vdW-DF-cx functional has recently been developed on this ground and has been shown to perform well for solids, layered materials, and for the S22 benchmark set. In particular the good results for lattice parameters and elastic response should be stressed. Here, our results for vdW-DF-cx demonstrate that this functional is capable of accurately describing the structure and properties of a wide range of systems; ranging from bulk oxides, to molecules adsorbed at surfaces and in porous media and for understanding the phase transition between a covalently bonded bulk solid to a dispersion bound layered material.

Given the adherences to various conservation rules and the associated potential for transferability, we believe that there are grounds for making some broader conclusions about the capability of the vdW-DF method. In fact, since the test cases can be seen as difficult, consisting of a range of problems where interactions compete, there is a potential for good performance also for general problems. At the very least, these results encourage us to test that conjecture in upcoming works. As such, in addition to providing a very competitive density functional for sparse matter, this work highlights the promise for further improving functionals, thanks to the robust and flexible formulation of the vdW-DF method.

\section{ACKNOWLEDGMENTS}

The authors thanks H. Rydberg and P. Erhart for useful discussions. Work by K.B., E.S., and P.H. was supported by the Swedish research council (VR) under grants VR-20114052 and VR-2010-4149 and by the Chalmers Area of Advance, Materials. Work by B.I.L. was supported via the pension from the Swedish pension system. T.T. acknowledges support from US NSF Grant No. DMR-1145968. V.R.C. was supported by the Materials Sciences and Engineering Division, Office of Basic Energy Sciences, U.S. Department of Energy. K.L. was supported by the U.S. Department of Energy, Office of Basic Energy Sciences, Division of Chemical Sciences, Geosciences, and Biosciences under award DEFG02-12ER16362. Portions of this work were performed at the Molecular Foundry, supported by the Office of Science, Office of Basic Energy Sciences, of the U.S. Department of Energy under Contract No. DEAC02-05CH11231. We are also grateful for allocation of computational resources by the Swedish National Infrastructure for Computing (SNIC) and by Wake Forest University.

\section{APPENDIX: FORMAL THEORY}

\section{The vdW-DF framework}

The vdW-DF framework is rooted in the ACF. The ACF embodies the fact that many-body interactions are reflected in the way a system responds to changes in the potential.

The ACF links the XC energy to the reducible and irreducible density-density correlation functions $\chi_{\lambda}(\omega)$ and $\tilde{\chi}_{\lambda}(\omega)$, respectively, at a given coupling constant $\lambda$-which, 
respectively, describes the induced charge given by an external and local potential-through an integral over the coupling-constant. Expressed as an integration over imaginary frequencies $u,{ }^{19,22,23,40}$ it reads

$$
E_{x c}+E_{\text {self }}=-\int_{0}^{1} d \lambda \int_{0}^{\infty} \frac{d u}{2 \pi} \operatorname{Tr}\left\{\chi_{\lambda}(i u) V\right\} .
$$

The infinite self-energy term $E_{\text {self }}$ cancels out a corresponding divergence in the right-hand side of Eq. (A1).

In the vdW-DF framework, the ACF is recast ${ }^{12,44}$ to include the coupling-constant implicitly within an effective longitudinal dielectric function $\kappa_{\mathrm{ACF}}(\mathrm{i} u)$, as follows:

$$
E_{x c}+E_{\text {self }}=\int_{0}^{\infty} \frac{d u}{2 \pi} \operatorname{Tr}\left\{\ln \left(\kappa_{\mathrm{ACF}}(\mathrm{i} u)\right)\right\} .
$$

The effective dielectric function $\kappa_{\mathrm{ACF}}(\mathrm{i} u)$ is defined by a longitudinal projection of a scalar dielectric function $\kappa_{\mathrm{ACF}}(\mathrm{i} u)$ $=\nabla \epsilon(\mathrm{i} u) \cdot \nabla G$. We also define an effective (couplingconstant averaged) local field response $\tilde{\chi}_{\mathrm{ACF}}$ using $\kappa_{\mathrm{ACF}}(i u)$ $\equiv 1-V \tilde{\chi}_{\mathrm{ACF}}(i u)$.

In principle Eq. (A2) can be made exact. ${ }^{117}$ In practice, an approximate scheme for the scalar dielectric function $\epsilon$ is employed through a single plasmon-pole approximation for the plasmon propagator $S(\omega) \equiv 1-\epsilon^{-1}(\omega)$. The form of $S$ is designed to observe all known conservation laws for the plasmons. $^{3}$

\section{2. $\mathrm{XC}$ hole conservation}

The relation

$$
\begin{aligned}
E_{x c} & =\frac{1}{2} \int d \mathbf{r} n(\mathbf{r}) \int \mathrm{d} \mathbf{u} \frac{1}{4 \pi u} n_{x c}(\mathbf{r} ; \mathbf{u}) \\
& =\int_{0}^{\infty} \frac{d u}{2 \pi} \operatorname{Tr}\left\{\ln \left(\kappa_{\mathrm{ACF}}(\mathrm{i} u)\right)\right\}-E_{\text {self }}
\end{aligned}
$$

links $\kappa_{\mathrm{ACF}}$ to $E_{x c}(\mathbf{r})$ and hence to an integral over the $\mathrm{XC}$ hole $n_{x c}(\mathbf{r}, \mathbf{u})$.

The XC hole is conserved if

$$
\int d \mathbf{u} n_{x c}(\mathbf{r} ; \mathbf{u}) \equiv n_{x c}\left(\mathbf{r} ; \mathbf{q}^{\prime}=0\right)=-1 .
$$

This condition can be formulated:

$$
\begin{aligned}
0 & =\int_{0}^{1} d \lambda \chi_{\lambda}\left(\mathrm{i} u ; \mathbf{q}=0, \mathbf{q}^{\prime}\right) \\
& =\left\langle\mathbf{q}=0\left|\ln \left(\kappa_{\mathrm{ACF}}(\mathrm{i} u)\right) V^{-1}\right| \mathbf{q}^{\prime}\right\rangle \\
& =-\sum_{n=1}^{\infty} \frac{1}{n}\left\langle\mathbf{q}=0\left|\left(\tilde{\chi}_{\mathrm{ACF}}(\mathrm{i} u) V\right)^{n} V^{-1}\right| \mathbf{q}^{\prime}\right\rangle
\end{aligned}
$$

A sufficient condition for conservation is therefore

$$
\tilde{\chi}_{\mathrm{ACF}}\left(\mathrm{i} u, \mathbf{q}=0, \mathbf{q}^{\prime}\right) \equiv 0 .
$$

The longitudinal projection of $\epsilon$ allows us to express

$$
\tilde{\chi}_{\mathrm{ACF}}\left(\mathrm{i} u, \mathbf{q}, \mathbf{q}^{\prime}\right)=4 \pi \mathbf{q} \cdot \mathbf{q}^{\prime}\left\langle\mathbf{q}\left|(1-S)^{-1} S\right| \mathbf{q}\right\rangle \text {. }
$$

Since $S(z)$ remains finite and free of poles in the upper right quadrant of the complex plane, condition (A6) is fulfilled. Conservation of the $\mathrm{XC}$ hole is therefore inherent to the
$\mathrm{XC}$ description expressed in Eq. (A2) and follows by the principles discussed in Ref. 118.

\section{3. vdW-DF in practice}

Functionals designed within the vdW-DF framework do not rely directly on Eq. (A2). Taking inspiration from the analysis of the plasmon-based analysis of surface-energy corrections, $^{22,23}$ the vdW-DF method splits the total XC energy functional into semilocal and nonlocal contributions,

$$
E_{x c}^{\mathrm{vdW}-\mathrm{DF}}[n]=E_{x c}^{\mathrm{sl}}[n]+E_{c}^{\mathrm{nl}}[n] .
$$

Several different exchange functionals have been suggested for the exchange part of $E_{x c}^{\mathrm{sl}}[n]$ as detailed in the main text. Only the LDA part of the correlation is included to avoid double counting semilocal correlation effects.

The vdW-DF method also considers GGA-type XC holes $^{3,11,12,44}$ defined by an inner functional

$$
E_{x c}^{0, i}+E_{\text {self }}=\int_{0}^{\infty} \frac{d u}{2 \pi} \operatorname{Tr}\{\ln (\epsilon(\mathrm{i} u))\},
$$

that is suggested by the GGA tradition. ${ }^{21-29,71,110}$ A specific functional is selected to describe $E_{x c}^{0, i}$ which in turn introduces a local parameter in $S$. Subtracting this term off the one in Eq. (A1), we obtain the nonlocal correlation energy

$$
E_{c}^{\mathrm{nl}}=\int_{0}^{\infty} \frac{d u}{2 \pi}\left[\operatorname{Tr} \ln \left(\kappa_{\mathrm{ACF}}(\mathrm{i} u)\right)-\operatorname{Tr} \ln (\epsilon(\mathrm{i} u))\right] .
$$

In the general geometry versions of vdW-DF, this term is further expanded to second order in $S$.

In general $E_{x c}^{\mathrm{sl}} \neq E_{x c}^{0, i}$ and the vdW-DF versions formally approximate the $\mathrm{XC}$ energy:

$$
\begin{gathered}
E_{x c}[n]=E_{x c}^{\mathrm{vdW}-\mathrm{DF}}[n]+\delta E_{x c}[n], \\
\delta E_{x c}=E_{x c}^{0, i}-E_{x c}^{\mathrm{sl}} .
\end{gathered}
$$

The mismatch is justified by the fact that $S$ is designed both with the aim of fulfilling formal constraints as well as to make $E_{c}^{\mathrm{nl}}$ simple to implement. Because of its simple form, the inner XC hole of vdW-DF does not capture short-range $\mathrm{XC}$ effects at the same level of sophistication as numerical GGA. ${ }^{29,44}$ However, this mismatch has the consequence that the automatic XC hole conservation secured by starting directly from Eq. (A2) is lost. vdW-DF1-cx is designed by using an exchange functional for $E_{x c}^{\mathrm{sl}}$ that makes the semilocal term resemble $E_{x c}^{0, i}$ as closely as feasible. This version largely restores the automatic conservation of the $\mathrm{XC}$ hole as detailed in Refs. 35 and 44.

${ }^{1}$ S. Grimme, J. Comput. Chem. 25, 1463 (2004).

${ }^{2}$ A. Tkatchenko and M. Scheffler, Phys. Rev. Lett. 102, 073005 (2009).

${ }^{3}$ M. Dion, H. Rydberg, E. Schröder, D. C. Langreth, and B. I. Lundqvist, Phys. Rev. Lett. 92, 246401 (2004).

${ }^{4}$ O. A. Vydrov and T. V. Voorhis, J. Chem. Phys. 133, 244103 (2010).

${ }^{5}$ K. Lee, È. D. Murray, L. Kong, B. I. Lundqvist, and D. C. Langreth, Phys. Rev. B 82, 081101 (2010).

${ }^{6}$ T. Thonhauser, V. R. Cooper, S. Li, A. Puzder, P. Hyldgaard, and D. C. Langreth, Phys. Rev. B 76, 125112 (2007).

${ }^{7}$ D. C. Langreth, B. I. Lundqvist, S. D. Chakarova-Käck, V. R. Cooper, M. Dion, P. Hyldgaard, A. Kelkkanen, J. Kleis, L. Kong, S. Li, P. G. Moses, E. Murray, A. Puzder, H. Rydberg, E. Schröder, and T. Thonhauser, J. Phys.: Condens. Matter 21, 084203 (2009). 
${ }^{8}$ Y. Andersson, D. C. Langreth, and B. I. Lundqvist, Phys. Rev. Lett. 76, 102 (1996).

${ }^{9}$ E. Hult, Y. Andersson, B. I. Lundqvist, and D. C. Langreth, Phys. Rev. Lett. 77, 2029 (1996).

${ }^{10}$ E. Hult, H. Rydberg, B. I. Lundqvist, and D. C. Langreth, Phys. Rev. B 59, 4708 (1999).

${ }^{11}$ H. Rydberg, B. I. Lundqvist, D. C. Langreth, and M. Dion, Phys. Rev. B 62, 6997 (2000).

${ }^{12}$ H. Rydberg, "Nonlocal correlations in density functional theory," Ph.D. thesis, Chalmers University of Technology, 2001.

${ }^{13}$ H. Rydberg, M. Dion, N. Jacobson, E. Schröder, P. Hyldgaard, S. I. Simak, D. C. Langreth, and B. I. Lundqvist, Phys. Rev. Lett. 91, 126402 (2003).

${ }^{14}$ M. Dion, "van der Waals forces in density functional theory," Ph.D. thesis, Rutgers University, 2004.

${ }^{15}$ D. C. Langreth, M. Dion, H. Rydberg, E. Schröder, P. Hyldgaard, and B. I. Lundqvist, Int. J. Quantum Chem. 101, 599 (2005).

${ }^{16}$ R. Eisenschitz and F. London, Z. Phys. 60, 491 (1930).

${ }^{17}$ F. London, Z. Phys. 63, 245 (1930).

${ }^{18}$ L. Hedin and B. I. Lundqvist, J. Phys. C 4, 2064 (1971).

${ }^{19}$ O. Gunnarsson and B. I. Lundqvist, Phys. Rev. B 13, 4274 (1976).

${ }^{20}$ J. P. Perdew and Y. Wang, Phys. Rev. B 45, 13244 (1992).

${ }^{21}$ M. Rasolt and D. J. W. Geldart, Phys. Rev. Lett. 35, 1234 (1975).

${ }^{22}$ D. C. Langreth and J. P. Perdew, Solid State Commun. 17, 1425 (1975).

${ }^{23}$ D. C. Langreth and J. P. Perdew, Phys. Rev. B 15, 2884 (1977).

${ }^{24}$ D. C. Langreth and M. J. Mehl, Phys. Rev. Lett. 47, 446 (1981).

${ }^{25}$ D. C. Langreth and S. H. Vosko, Phys. Rev. Lett. 59, 497 (1987).

${ }^{26}$ D. C. Langreth and S. H. Vosko, Adv. Quantum Chem. 21, 175 (1990).

${ }^{27}$ J. P. Perdew and Y. Wang, Phys. Rev. B 33, 8800 (1986).

${ }^{28}$ J. P. Perdew, K. Burke, and M. Ernzerhof, Phys. Rev. Lett. 77, 3865 (1996).

${ }^{29}$ J. P. Perdew, K. Burke, and Y. Wang, Phys. Rev. B 54, 16533 (1996).

${ }^{30}$ Y. Zhang and W. Yang, Phys. Rev. Lett. 80, 890 (1998).

${ }^{31}$ T. Thonhauser, A. Puzder, and D. C. Langreth, J. Chem. Phys. 124, 164106 (2006).

${ }^{32}$ É. D. Murray, K. Lee, and D. C. Langreth, J. Chem. Theory Comput. 5, 2754 (2009).

${ }^{33}$ V. R. Cooper, Phys. Rev. B 81, 161104 (2010).

${ }^{34}$ J. Klimeš, D. R. Bowler, and A. Michaelides, J. Phys.: Condens. Matter 22, 022201 (2010).

${ }^{35}$ K. Berland and P. Hyldgaard, Phys. Rev. B 89, 035412 (2014).

${ }^{36}$ P. Jurečka, J. Šponer, J. Černý, and P. Hobza, Phys. Chem. Chem. Phys. 8, 1985 (2006).

${ }^{37}$ N. Bohr, J. Lindhard, and K. Dan, Vidensk. Selsk. Mat. Fys. Medd. 28(7), 1 (1954).

${ }^{38}$ J. Lindhard and K. Dan, Vidensk. Selsk. Mat. Fys. Medd. 28(8), 1 (1954).

${ }^{39}$ K. Sawada, Phys. Rev. 106, 372 (1957).

${ }^{40} \mathrm{D}$. Pines and P. Nozières, The Theory of Quantum Liquids (AddisonWesley publishing Company, Inc, Redwood City, California, 1989), Vol. I.

${ }^{41}$ B. I. Lundqvist, Phys. Kondens. Materie 6, 193 (1967).

${ }^{42}$ B. I. Lundqvist, Phys. Kondens. Materie 6, 206 (1967).

${ }^{43}$ B. I. Lundqvist, Phys. Kondens. Materie 7, 117 (1968).

${ }^{44}$ P. Hyldgaard, K. Berland, and E. Schröder, "Adiabatic connection and response in van der Waals density functionals" (unpublished).

${ }^{45}$ K. Berland and P. Hyldgaard, Phys. Rev. B 87, 205421 (2013).

${ }^{46}$ J. Kleis, E. Schröder, and P. Hyldgaard, Phys. Rev. B 77, 205422 (2008).

${ }^{47}$ O. Gunnarsson, M. Jonson, and B. I. Lundqvist, Phys. Rev. B 20, 3136 (1979).

${ }^{48}$ E. Ziambaras, J. Kleis, E. Schröder, and P. Hyldgaard, Phys. Rev. B 76, 155425 (2007).

${ }^{49}$ P. Lazić, N. Atodiresei, V. Caciuc, R. Brako, B. Gumhalter, and S. Blügel, J. Phys.: Condens. Matter 24, 424215 (2012).

${ }^{50}$ J. Klimeš, D. R. Bowler, and A. Michaelides, Phys. Rev. B 83, 195131 (2011).

${ }^{51}$ K. Rapcewicz and N. W. Ashcroft, Phys. Rev. B 44, 4032 (1991).

${ }^{52}$ K. Burke, J. Chem. Phys. 136, 150901 (2012).

${ }^{53} \mathrm{In}$ addition to the layered-system results presented in Ref. 35 we have added new results for $\mathrm{C}_{8} \mathrm{~K}$ (also reported in Table III) and for $\mathrm{Bi}_{2} \mathrm{Te}_{3}$. The latter was obtained with normconserving pseudopotentials; fully relaxing both the forces and stresses. Our self-consistent vdW-DF1/vdWDF2/vdW-DF-C09/vdW-DF-cx calculations determine in-plane lattice constants for $\mathrm{Bi}_{2} \mathrm{Te}_{3} a=4.47 / 4.55 / 4.35 / 4.36 \AA$, out-of-plane lattice constant $c=11.03 / 10.85 / 10.08 / 10.13$, and elastic constants $C_{33}$ $=19 / 28 / 48 / 43 \mathrm{GPa}$, respectively. Corresponding experimental lattice con- stants are $a_{0}=4.39 \AA, c_{0}=10.166 \AA$, Ref. 119. An experimental elastic constant $C_{33}^{0}=47.7 \mathrm{GPa}$ is listed in the comparison in Ref. 108 .

${ }^{54}$ A. Kraft, R. Temirov, S. K. M. Henze, S. Soubatch, M. Rohlfing, and F. S. Tautz, Phys. Rev. B 74, 041402 (2006).

${ }^{55}$ R. Sabatini, E. Kucukbenli, B. Kolb, T. Thonhauser, and S. de Gironcoli, J. Phys.: Condens. Matter 24, 424209 (2012).

${ }^{56}$ Y. Yao, N. Nijem, J. Li, Y. J. Chabal, D. C. Langreth, and T. Thonhauser, Phys. Rev. B 85, 064302 (2012).

${ }^{57}$ Y. Kuroiwa, S. Aoyagi, A. Sawada, J. Harada, E. Nishibori, M. Takata, and M. Sakata, Phys. Rev. Lett. 87, 217601 (2001).

${ }^{58}$ V. G. Gavrilyachenko, R. I. Spinko, M. A. Martynenko, and E. G. Fesenko, Sov. Phys. Solid State 12, 1203 (1970).

${ }^{59}$ Z. Li, M. Grimsditch, C. M. Foster, and S.-K. Chan, J. Phys. Chem. Solids 57, 1433 (1996).

${ }^{60}$ R. D. King-Smith and D. Vanderbilt, Phys. Rev. B 49, 5828 (1994).

${ }^{61}$ U. V. Waghmare and K. M. Rabe, Phys. Rev. B 55, 6161 (1997).

${ }^{62}$ H. Y. Hwang, Y. Iwasa, M. Kawasaki, B. Keimer, N. Nagaosa, and Y. Tokura, Nature Mater. 11, 103 (2012).

${ }^{63}$ S. V. Kalinin and N. A. Spaldin, Science 341, 858 (2013).

${ }^{64}$ W. Zhong, R. D. King-Smith, and D. Vanderbilt, Phys. Rev. Lett. 72, 3618 (1994).

${ }^{65}$ Z. Wu and R. E. Cohen, Phys. Rev. Lett. 95, 037601 (2005).

${ }^{66}$ Z. Wu, R. E. Cohen, and D. J. Singh, Phys. Rev. B 70, 104112 (2004).

${ }^{67}$ W. Zhong, D. Vanderbilt, and K. M. Rabe, Phys. Rev. Lett. 73, 1861 (1994).

${ }^{68}$ W. Zhong, D. Vanderbilt, and K. M. Rabe, Phys. Rev. B 52, 6301 (1995).

${ }^{69}$ R. Armiento and A. E. Mattsson, Phys. Rev. B 72, 085108 (2005).

${ }^{70} \mathrm{Z}$. Wu and R. E. Cohen, Phys. Rev. B 73, 235116 (2006).

${ }^{71}$ J. P. Perdew, A. Ruzsinszky, G. I. Csonka, O. A. Vydrov, G. E. Scuseria, L. A. Constantin, X. Zhou, and K. Burke, Phys. Rev. Lett. 100, 136406 (2008).

${ }^{72}$ H. Furukawa, K. E. Cordova, M. O'Keeffe, and O. M. Yaghi, Science 341, 974 (2013).

${ }^{73}$ D. J. Tranchemontagne, K. S. Park, H. Furukawa, J. Eckert, C. B. Knobler, and O. M. Yaghi, J. Phys. Chem. C 116, 13143 (2012).

${ }^{74}$ H.-C. Zhou, J. R. Long, and O. M. Yaghi, Chem. Rev. 112, 673 (2012).

${ }^{75}$ P. Canepa, C. A. Arter, E. M. Conwill, D. H. Johnson, B. A. Shoemaker, K. Z. Soliman, and T. Thonhauser, J. Mater. Chem. A 1, 13597 (2013).

${ }^{76}$ P. Canepa, N. Nijem, Y. Chabal, and T. Thonhauser, Phys. Rev. Lett. 110, 026102 (2013).

${ }^{77}$ W. Zhou, H. Wu, and T. Yildirim, J. Am. Chem. Soc. 130, 15268 (2008).

${ }^{78}$ J. Park, H. Kim, S. S. Han, and Y. Jung, J. Phys. Chem. Lett. 3, 826 (2012).

${ }^{79}$ W. L. Queen, C. M. Brown, D. K. Britt, P. Zajdel, M. R. Hudson, and O. M. Yaghi, J. Phys. Chem. C 115, 24915 (2011).

${ }^{80}$ Y. Baskin and Y. Meyer, Phys. Rev. 100, 544 (1955).

${ }^{81}$ F. P. Bundy, H. M. Strong, H. P. Bovenbeck, and R. H. Wentorf, J. Chem. Phys. 35, 383 (1961).

${ }^{82}$ R. Z. Khaliullin, H. Eshet, T. D. Kühne, J. Behler, and M. Parrinello, Nature Mater. 10, 693 (2011).

${ }^{83}$ R. Berman and F. Simon, Z. Elektrochem. 59, 333 (1955).

${ }^{84}$ R. Zacharia, H. Ulbricht, and T. Hertel, Phys. Rev. B 69, 155406 (2004).

${ }^{85}$ D. E. Nixon and G. S. Parry, J. Phys. C 2, 1732 (1969).

${ }^{86}$ M. S. Dresselhaus and G. Dresselhaus, Adv. Phys. 30, 139 (1981).

${ }^{87}$ S. Aronson, F. J. Salzano, and D. Ballafio, J. Chem. Phys. 49, 434 (1968).

${ }^{88}$ K. Berland, T. L. Einstein, and P. Hyldgaard, Phys. Rev. B 80, 155431 (2009).

${ }^{89}$ M. Mura, A. Gulans, T. Thonhauser, and L. Kantorovich, Phys. Chem. Chem. Phys. 12, 4759 (2010)

${ }^{90}$ G. Li, I. Tamblyn, H.-J. Gao, V. R. Cooper, and J. B. Neaton, Phys. Rev. B 85, 121409 (2012).

${ }^{91}$ K. Lee, K. Berland, M. Yoon, S. Andersson, E. Schröder, P. Hyldgaard, and B. I. Lundqvist, J. Phys: Condens. Matter 24, 424213 (2012).

${ }^{92}$ K. Glckler, C. Seidel, A. Soukopp, M. Sokolowski, E. Umbach, M. Bhringer, R. Berndt, and W.-D. Schneider, Surf. Sci. 405, 1 (1998).

${ }^{93}$ M. Eremtchenko, J. A. Schaefer, and F. S. Tautz, Nature (London) 425, 602 (2003)

${ }^{94}$ S. Sachs, C. H. Schwalb, M. Marks, A. Schoell, F. Reinert, E. Umbach, and U. Hoefer, J. Chem. Phys. 131, 144701 (2009).

${ }^{95}$ L. Romaner, D. Nabok, P. Puschnig, E. Zojer, and C. Ambrosch-Draxl, New J. Phys. 11, 053010 (2009).

${ }^{96}$ J. Björk, F. Hanke, C.-A. Palma, P. Samori, M. Cecchini, and M. Persson, J. Phys. Chem. Lett. 1, 3407 (2010).

${ }^{97}$ A. Tkatchenko, L. Romaner, O. T. Hofmann, E. Zojer, C. AmbroschDraxl, and M. Scheffler, MRS Bull. 35, 435 (2010). 
${ }^{98}$ V. G. Ruiz, W. Liu, E. Zojer, M. Scheffler, and A. Tkatchenko, Phys. Rev. Lett. 108, 146103 (2012)

${ }^{99}$ E. Umbach, M. Sokolowski, and R. Fink, Appl. Phys. A 63, 565 (1996).

${ }^{100}$ U. Stahl, D. Gador, A. Soukopp, R. Fink, and E. Umbach, Surf. Sci. 414, 423 (1998).

${ }^{101}$ K. A. Fichthorn and R. A. Miron, Phys. Rev. Lett. 89, 196103 (2002).

${ }^{102}$ The average heights of the anhydride oxygen atoms, carbon atoms, and carboxyl oxygen atoms in the adsorbed PTCDA molecule are $3.09 \AA$, $3.06 \AA$, and $2.97 \AA$, respectively. In comparison with the experimental values of $2.97 \AA, 2.86 \AA$, and $2.68 \AA$, they are overestimated by $0.12 \AA$, $0.20 \AA$, and $0.29 \AA$, respectively. Including relaxations for the bare surface, the first atomic layer of the surface moves away from the substrate by $0.020 \AA$ on average. After adsorption, however, the layer is pushed toward the surface by $0.016 \AA$ on average. The buckling of the surface, measured as the difference between the minimum and the maximum vertical displacement of the atoms is $0.09 \AA$ as compared to $0.18 \AA$ for the molecule.

${ }^{103}$ H. Yildirim, T. Greber, and A. Kara, J. Phys. Chem. C 117, 20572 (2013).

${ }^{104}$ J. Lüder, B. Sanyal, O. Eriksson, C. Puglia, and B. Brena, Phys. Rev. B 89, 045416 (2014).

${ }^{105}$ J. Carrasco, W. Liu, A. Michaelides, and A. Tkatchenko, J. Chem. Phys. 140, 084704 (2014).
${ }^{106}$ X. Wu, M. C. Vargas, S. Nayak, V. Lotrich, and G. Scoles, J. Chem. Phys. $115,8748(2001)$

${ }^{107}$ S. D. Chakarova and E. Schröder, J. Chem. Phys. 122, 054102 (2005).

${ }^{108}$ T. Björkman, A. Gulans, A. V. Krasheninnikov, and R. M. Nieminen, J. Phys.: Condens. Matter 24, 424218 (2012).

${ }^{109}$ K. Berland, Ø. Borck, and P. Hyldgaard, Comput. Phys. Commun. 182, 1800 (2011).

${ }^{110}$ V. N. Staroverov, G. E. Scuseria, J. Tao, and J. P. Perdew, Phys. Rev. B 69, 075102 (2004).

${ }^{111}$ J. Schwinger, Phys. Rev. A 22, 1827 (1980).

${ }^{112}$ P. Elliott and K. Burke, Can. J. Chem. 87, 1485 (2009).

${ }^{113}$ J. P. Perdew, L. A. Constantin, E. Sagvolden, and K. Burke, Phys. Rev. Lett. 97, 223002 (2006).

${ }^{114}$ A. D. Becke, Phys. Rev. A 38, 3098 (1988).

${ }^{115}$ F. O. Kannemann and A. D. Becke, J. Chem. Theory Comput. 5, 719 (2009).

${ }^{116}$ K. Lee, A. K. Kelkkanen, K. Berland, S. Andersson, D. C. Langreth, E. Schröder, B. I. Lundqvist, and P. Hyldgaard, Phys. Rev. B 84, 193408 (2011).

${ }^{117}$ One can select a scalar, nonlocal dielectric constant $\epsilon(i u)$ that satisfies ${ }^{12}$ Eq. (A2) for any given $E_{x c}$.

${ }^{118}$ J. F. Dobson and B. P. Dinte, Phys. Rev. Lett. 76, 1780 (1996).

${ }^{119}$ S. Nakajima, J. Phys. Chem. Solids 24, 479 (1963). 